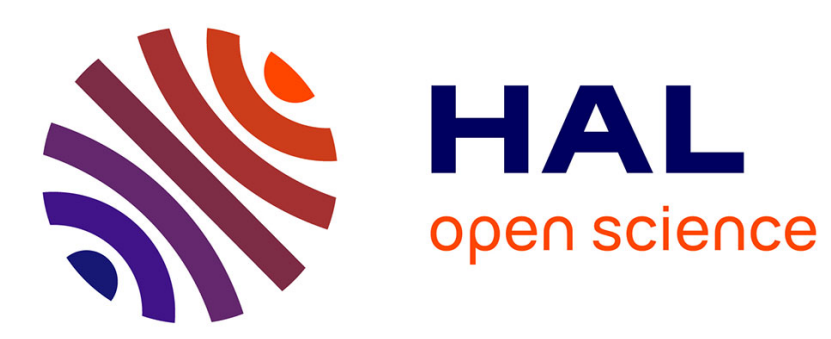

\title{
Effect of the Casimir-Polder force on the collective oscillations of a trapped Bose-Einstein condensate
}

Mauro Antezza, Lev P. Pitaevskii, Sandro Stringari

\section{To cite this version:}

Mauro Antezza, Lev P. Pitaevskii, Sandro Stringari. Effect of the Casimir-Polder force on the collective oscillations of a trapped Bose-Einstein condensate. Physical Review A : Atomic, molecular, and optical physics [1990-2015], 2004, 70 (5), pp.053619. 10.1103/PhysRevA.70.053619 . hal-02960126

\section{HAL Id: hal-02960126 \\ https://hal.science/hal-02960126}

Submitted on 7 Oct 2020

HAL is a multi-disciplinary open access archive for the deposit and dissemination of scientific research documents, whether they are published or not. The documents may come from teaching and research institutions in France or abroad, or from public or private research centers.
L'archive ouverte pluridisciplinaire HAL, est destinée au dépôt et à la diffusion de documents scientifiques de niveau recherche, publiés ou non, émanant des établissements d'enseignement et de recherche français ou étrangers, des laboratoires publics ou privés. 


\title{
Effect of the Casimir-Polder force on the collective oscillations of a trapped Bose-Einstein condensate
}

\author{
Mauro Antezza, ${ }^{1, *}$ Lev P. Pitaevskii, ${ }^{1,2}$ and Sandro Stringari ${ }^{1}$ \\ ${ }^{1}$ Dipartimento di Fisica, Università di Trento and Istituto Nazionale per la Fisica della Materia INFM-BEC, \\ I-38050 Povo, Trento, Italy \\ ${ }^{2}$ Kapitza Institute for Physical Problems, ul. Kosygina 2, 117334 Moskow, Russia
}

(Received 19 July 2004; published 24 November 2004; publisher error corrected 2 December 2004)

\begin{abstract}
We calculate the effect of the interaction between an optically active material and a Bose-Einstein condensate on the collective oscillations of the condensate. We provide explicit expressions for the frequency shift of the center-of-mass oscillation in terms of the potential generated by the substrate and of the density profile of the gas. The form of the potential is discussed in detail and various regimes (van der Waals-London, CasimirPolder, and thermal regimes) are identified as a function of the distance of atoms from the surface. Numerical results for the frequency shifts are given for the case of a sapphire dielectric substrate interacting with a harmonically trapped condensate of ${ }^{87} \mathrm{Rb}$ atoms. We find that at distances of 4-8 $\mu \mathrm{m}$, where thermal effects become visible, the relative frequency shifts produced by the substrate are of the order $10^{-4}$ and hence accessible experimentally. The effects of nonlinearities due to the finite amplitude of the oscillation are explicitly discussed. Predictions are also given for the radial breathing mode.
\end{abstract}

DOI: 10.1103/PhysRevA.70.053619 PACS number(s): 03.75.Kk, 67.40.Db, 77.22. - d, 78.20.-e

\section{INTRODUCTION}

The study of the force felt by an atom near a surface has recently become a popular subject of research (see, for example, [1,2] and references therein) both in view of nanotechnological applications [3] and because of the possibility of investigating fundamental forces at the submicron scale [4]. These studies extend previous investigations of the generalized van der Waals forces between macroscopic bodies [5] to more microscopic systems. Experiments with BoseEinstein condensates near a surface [6-8] are very promising in this respect because of the high accuracy of the measurements achievable with ultracold gases.

Historically the interatomic forces were first introduced in 1873 by van der Waals in order to explain the deviations of the thermodynamic behavior of real gases from the ideal laws. Only in 1930 did London [9] provide a first quantitative description of the force. He applied second-order perturbation theory to the quantum Hamiltonian to evaluate the electrostatic interaction between two dipoles, induced by the fluctuation of the electromagnetic field. He succeeded in deriving the most famous power law $1 / d^{6}$, usually known as the van der Waals-London interaction, where $d$ is the interatomic distance. Almost 20 years later, in 1948, Casimir and Polder [10], taking into account relativistic retardation effects within fourth-order perturbation theory, found that, at large distances, the potential decays like $1 / d^{7}$ rather than like $1 / d^{6}$.

The existence of these attractive forces between neutral atoms gives rise to analogous forces between a single atom and a dielectric or a metallic wall, as well as to forces between macroscopic bodies separated by small distances [11]. Between the end of the 1950s and the beginning of the 1960s

*Electronic address: antezza@science.unitn.it
Lifshitz developed the general theory of the van der Waals forces $[12,13]$ for inhomogeneous media, taking into account the quantum fluctuations of the electromagnetic field and their perturbation produced by the media. The theory describes the interaction between continuous media in terms of their dielectric functions and of the temperature. The van der Waals-London and Casimir-Polder forces are recovered as limiting cases of the more general theory, which also accounts for the effects induced by the thermal fluctuations of the electromagnetic field. Thermal effects provide the asymptotic behavior of the interaction at distances much larger than the thermal wavelength of photons.

The purpose of the present work is to show that the study of the collective oscillations of a Bose-Einstein condensate of ultracold atoms can provide a useful probe of such forces. Bose-Einstein condensates are very dilute samples and consequently the force produced by the surface can be safely calculated starting from the interaction felt by the individual atoms in the condensate [11]. In this paper we will be mainly concerned with the center-of-mass oscillation of a condensate confined by a harmonic trap. The center-of-mass motion has the main advantage of being independent of two-body interactions and its frequency consequently provides an ideal probe of the additional force generated by the wall. The effect of the force on the frequency of the center-of-mass oscillation depends on the distance of the condensate from the surface and on the temperature of the surface as well as on the optical properties of the surface and of the atoms in the condensate. The effect also depends on the thickness of the condensate which in actual configurations can be of the same order as the distance from the wall. In some limiting cases, corresponding to short and large distances, it is possible to obtain analytic or semianalytic predictions that will be discussed systematically in our paper. They can serve as a natural guide for first quantitative estimates and as useful tests of more complete numerical approaches. 
The paper is organized as follows.

In Sec. II we derive some general results for the shift of the collective frequencies of a harmonically trapped BoseEinstein condensate subject to an additional weak external force.

In Sec. III we employ Lifshitz theory to calculate the force acting on a dilute atomic gas placed near a surface.

In Sec. IV we discuss the transition from the van der Waals-London potential, holding at very short distances from the surface and decaying like $1 / d^{3}$, to the CasimirPolder potential which accounts for relativistic retardation effects and holds at larger distances where it decays like $1 / d^{4}$.

In Sec. $\mathrm{V}$ we discuss the transition from the $1 / d^{4}$ law characterizing the Casimir-Polder potential to the behavior of the potential at much larger distances from the surface (larger than the thermal wavelength of photons) where it decays like $1 / d^{3}$ as a consequence of the thermal fluctuations of the electromagnetic field.

In Sec. VI we discuss the role of the optical properties of the interacting media with specific emphasis to the case of the dielectric sapphire $\left(\mathrm{Al}_{2} \mathrm{O}_{3}\right)$ substrate and of the rubidium atoms which form the condensate.

In Sec. VII we provide results for the shifts of the collective frequencies as a function of the distance of the condensate from the surface, calculated at different temperatures.

Finally, in the Appendix we provide results for the frequency shift of the radial compression mode in very elongated harmonic traps.

\section{CENTER-OF-MASS OSCILLATION OF A TRAPPED GAS IN THE PRESENCE OF A WEAK PERTURBATION}

After the first experimental realization of Bose-Einstein condensation in dilute vapors of alkali atoms the experimental and theoretical research in the field of ultacold quantum gases has grown in an impressive way (for general reviews see, for example, [14-17]). Bose-Einstein-condensed gases are dilute, ultracold samples characterized by unique properties of coherence and superfluidity. They give rise, among others, to interference phenomena $[18,19]$ as well as to a variety of collective oscillations [20-22]. The relatively high density of Bose-Einstein-condensed samples (compared to the one of noncondensed trapped gases), as well as the possibility of imaging the atomic cloud after expansion, where the measured sizes become macroscopically large, permit one to achieve very accurate determinations of the frequencies of the collective oscillations, thereby providing interesting opportunities for precision measurements.

In this section we will calculate the effect of a weak perturbation on the center-of-mass oscillation of a harmonically trapped gas. The frequency of this oscillation, in the absence of the perturbation, coincides with the frequency of the trap. This result, also known as the Kohn theorem [23], is independent of the amplitude of the oscillation, interatomic forces, and temperature as well as of the quantum nature of the sample. Deviations of the measured frequency from the oscillator value can then be used as a useful probe of addi-

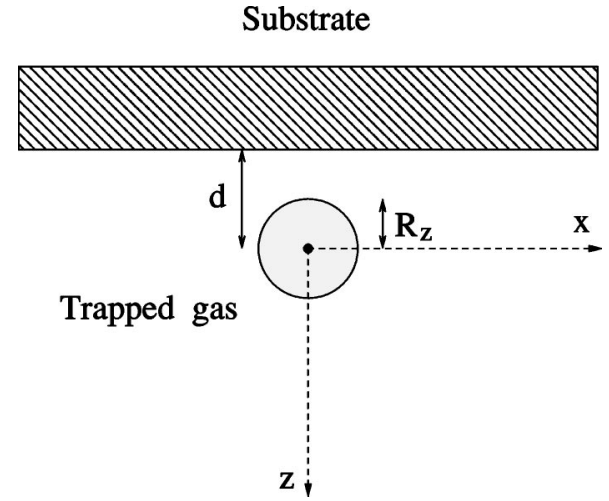

FIG. 1. Schematic figure of the substrate-trapped gas system. Gravity is oriented along $z$.

tional interactions, like the one generated by the surface. In Fig. 1 we show a schematic representation of the geometry considered in the present work. A dilute gas trapped by the harmonic potential

$$
V_{h o}(\mathbf{r})=\frac{m}{2} \omega_{x}^{2} x^{2}+\frac{m}{2} \omega_{y}^{2} y^{2}+\frac{m}{2} \omega_{z}^{2} z^{2}
$$

is placed close to an ideal wall generating a potential $V_{\text {surf }}(z)$ so that the full potential felt by each atom is given by

$$
V(\mathbf{r})=V_{h o}(\mathbf{r})+V_{\text {surf }}(z) .
$$

In the following we will calculate the changes in the frequency of the center-of-mass oscillation along the $z$ th direction caused by the presence of the surface. In the Appendix we provide results also for the radial breathing mode in the case of very elongated axisymmetric traps $\left(\omega_{y} \ll \omega_{x}=\omega_{z}\right)$.

The equations for the center-of-mass (c.m.) coordinate $Z_{\text {c.m. }}=(1 / N)\left\langle\sum_{i=1}^{N} z_{i}\right\rangle$ and the total momentum $P_{z}=\left\langle\sum_{i=1}^{N} p_{z_{i}}\right\rangle$ along the $z$ th direction ( $N$ is the number of atoms of the trapped gas) can be written in the general form

$$
\frac{d Z_{\text {c.m. }}}{d t}=\frac{P_{z}}{N m}
$$

and

$$
\begin{aligned}
\frac{1}{N} \frac{d P_{z}}{d t} & =-\frac{1}{N}\left\langle\sum_{i=1}^{N} \partial_{z_{i}} V\left(\mathbf{r}_{i}\right)\right\rangle \\
& =-m \omega_{z}^{2} Z_{\text {c.m. }}-\int n(\mathbf{r}, t) \partial_{z} V_{\text {surf }}(z) d \mathbf{r},
\end{aligned}
$$

where we have used expression (1) for $V_{h o}$ and we have expressed the average of the external force $\partial_{z} V_{\text {surf }}$ in terms of the density $n$ of the gas here assumed to be normalized to unity $\left(\int d \mathbf{r} n=1\right)$. Notice that the equation for the total momentum is not affected by the two-body potential. In the absence of the perturbation $\left(V_{\text {surf }}=0\right)$ one recovers the equations of the harmonic oscillator and the center-of-mass oscillation corresponds to a rigid oscillation of the density. The effect of the perturbation $V_{\text {surf }}$ can then be calculated, in first-order perturbation theory, using the scaling ansatz $n(\mathbf{r}, t)=n_{0}\left(x, y, z-Z_{\text {c.m. }}(t)\right)$ in Eq. (4), where $n_{0}$ is the equi- 
librium density of the gas. The equation for the center-ofmass coordinate finally takes the simple form

$$
\frac{d^{2} Z_{\text {c.m. }}}{d t^{2}}=-\omega_{z}^{2} Z_{\text {c.m. }}-\frac{1}{m} \int n_{0}(\mathbf{r}) \partial_{z} V_{\text {surf }}\left(z+Z_{\text {c.m. }}\right) d \mathbf{r},
$$

where, in the integral, we have made the substitution $(z$ $-Z_{\text {c.m. }}$ ) $\rightarrow z$. Equations (3) and (5) have now a closed form. In the limit of small oscillations we can expand the perturbation term up to terms linear in $Z_{\mathrm{c} . \mathrm{m} .}(t)$ and the equation for the center of mass takes again a harmonic form with the renormalized oscillator frequency

$$
\omega_{\mathrm{c} . \mathrm{m} .}^{2}=\omega_{z}^{2}+\frac{1}{m} \int n_{0}(\mathbf{r}) \partial_{z}^{2} V_{\text {surf }}(z) d \mathbf{r} .
$$

It is worth stressing that the result (6) is exact up to firstorder corrections in $V_{\text {surf }}$ and holds independently of the quantum or classical nature of the gas which instead determines the actual form of the density profile $n_{0}$. Equation (6) can be further simplified by introducing the so-called onedimensional (1D) column density

$$
n_{0}^{z}(z)=\int n_{0}(x, y, z) d x d y
$$

and using the fact that $V_{\text {surf }}$ depends only on the zth coordinate. One then finds the result

$$
\omega_{\mathrm{c} . \mathrm{m} .}^{2}=\omega_{z}^{2}+\frac{1}{m} \int_{-R_{z}}^{+R_{z}} n_{0}^{z}(z) \frac{d^{2} V_{\text {surf }}(z)}{d z^{2}} d z .
$$

Equation (8) shows that the ingredients needed to calculate the effect of the perturbation on the shift of the center-of- mass frequency are the explicit form of the potential $V_{\text {surf }}$, which will be discussed in the following sections, and the 1D column density (7). For a Bose-Einstein condensate in its ground state the density profile is easily evaluated in the so-called Thomas-Fermi approximation. This approximation holds for large condensates where, for gases interacting with positive scattering length, the kinetic energy of the trapped condensate can be neglected and one finds the analytic result [14] $n_{0}(x, y, z)=g^{-1}\left[\mu-V_{h o}(\mathbf{r})\right]$ when $n_{0} \geqslant 0$ and 0 elsewhere. Here $\mu=\left(\hbar \omega_{h o} / 2\right)\left(15 N a / a_{h o}\right)^{2 / 5}$ is the chemical potential, $N$ is the number of atoms, $\omega_{h o}=\left(\omega_{x} \omega_{y} \omega_{z}\right)^{1 / 3}$ is the geometrical average of the three trap frequencies, $a_{h o}$ $=\sqrt{\hbar /\left(m \omega_{h o}\right)}$ is the oscillator length, and $g=4 \pi \hbar^{2} a / m$ is the interaction coupling constant fixed by the $s$-wave scattering length $a$. Integration of $n_{0}$ with respect to $x$ and $y$ yields

$$
n_{0}^{z}(z)=\frac{15}{16} \frac{1}{R_{z}}\left(1-\frac{z^{2}}{R_{z}^{2}}\right)^{2},
$$

where $R_{z}$ is the Thomas-Fermi radius in the $z$ th direction, fixed by the relation $\mu=m \omega_{z}^{2} R_{z}^{2} / 2$. Typical values for $R_{z}$ in standard experimental conditions can be of the order of a few microns [25].

Equations (8) and (9) are the key result of this section and will be used in the second part of the paper to calculate the effect of the force produced by the surface on the frequency shift of the center-of-mass oscillation. The integral (8) should be in general carried out numerically. There are, however, important cases where the integration can be found analytically. This is the case, for example, if one can approximate the potential with the algebraic decay $V^{(n)}(z)=-a_{n} /(d+z)^{n}$ where $d$ is the distance between the wall and the center of the harmonic trap [24]. In this case we find the result

$$
\omega_{\mathrm{c} . \mathrm{m} .}^{2}=\omega_{z}^{2}-\frac{20 a_{4}}{m d^{6}} \frac{1}{\left(1-\chi^{2}\right)^{3}}
$$

and

$$
\omega_{\mathrm{c.m.}}^{2}=\omega_{z}^{2}-\frac{15 a_{3}}{4 m d^{5}} \frac{10 \chi^{3}-6 \chi-3\left(1-\chi^{2}\right)^{2} \ln [(1-\chi) /(1+\chi)]}{\left(1-\chi^{2}\right)^{2} \chi^{5}}
$$

for the most relevant $n=4$ and $n=3$ cases, respectively, where $\chi \equiv R_{z} / d$. Results (10) and (11) explicitly point out the role played by the finite size of the condensate. In particular, only in the limit $R_{z} \ll d(\chi \ll 1)$ will the above equations approach the power law behaviors $1 / d^{6}$ and $1 / d^{5}$ for the frequency shift expected for a pointlike condensate.

The above results have been derived in the linear regime. If the amplitude of the oscillation is comparable to the distance from the surface, nonlinear effects become important and modify the value of the frequency shift. Nonlinear effects result in a frequency shift as well as in the occurrence of higher harmonics. They can be calculated starting from the general Eqs. (3) and (5) for the center-of-mass coordinate. Notice that, due to the harmonic nature of $V_{h o}$, both effects are absent if $V_{\text {surf }}=0$. Due to the perturbative nature of the problem, we can use the periodic law $Z_{\text {c.m. }}$. $=a \cos \left(\omega_{\mathrm{c} . \mathrm{m} .} t\right)$ to evaluate the integral of Eq. (5), where $a$ is the amplitude of the oscillation. One can then introduce the periodic function

$$
Q(t) \equiv \frac{1}{m} \int n_{0}^{z}(z) \partial_{z} V_{\text {surf }}\left(z+a \cos \left(\omega_{\mathrm{c} . \mathrm{m} .} t\right)\right) d z
$$

which can be conveniently expanded in a Fourier series. For the calculation of the frequency shift only the term propor- 
tional to $\cos \left(\omega_{\text {c.m. }} t\right)$ is important and one can write

$$
\omega_{\text {c.m. }}^{2}-\omega_{z}^{2}=\frac{\omega_{\text {c.m. } .}}{\pi a} \int_{0}^{2 \pi / \omega_{\text {c.m. }}} Q(t) \cos \left(\omega_{\text {c.m. }} t\right) d t .
$$

Expansion of Eq. (13) up to terms quadratic in $a$ and use of the 1D column density finally yield the result

$$
\begin{aligned}
\omega_{\mathrm{c} . \mathrm{m} .}^{2}-\omega_{z}^{2}= & \frac{1}{m} \int_{-R_{z}}^{R_{z}} n_{0}^{z}(z) \frac{d^{2} V_{\text {surf }}(z)}{d z^{2}} d z \\
& +\frac{a^{2}}{8 m} \int_{-R_{z}}^{R_{z}} n_{0}^{z}(z) \frac{d^{4} V_{\text {surf }}(z)}{d z^{4}} d z
\end{aligned}
$$

for the frequency shift which generalizes Eq. (8) by including the nonlinear correction in $a^{2}$.

\section{FORCE BETWEEN THE SURFACE AND A SINGLE ATOM}

The interaction produced by a surface on a single atom includes, in its general form, nontrivial relativistic effects as well as quantum and thermal fluctuations of the field. The force can be calculated starting from the general theory developed by Lifshitz for the free energy associated with inhomogeneous media. For two infinite plates separated by a distance $d$ and in thermal equilibrium with the thermal radiation at temperature $T$, the force $F_{\text {surf }}$ per unit surface can be written as [13]

$$
\begin{aligned}
F_{\text {surf }}(d)= & \frac{k_{B} T}{16 \pi d^{3}} \int_{0}^{\infty} x^{2}\left[\frac{\left(\varepsilon_{10}+1\right)\left(\varepsilon_{20}+1\right)}{\left(\varepsilon_{10}-1\right)\left(\varepsilon_{20}-1\right)} e^{x}-1\right]^{-1} d x+\frac{k_{B} T}{\pi c^{3}} \sum_{n=1}^{\infty} \xi_{n}^{3} \int_{1}^{\infty} p^{2}\left\{\left[\frac{\left(s_{1}+p\right)\left(s_{2}+p\right)}{\left(s_{1}-p\right)\left(s_{2}-p\right)} \exp \left(\frac{2 p \xi_{n} d}{c}\right)-1\right]^{-1}\right. \\
& \left.+\left[\frac{\left(s_{1}+p \varepsilon_{1}\right)\left(s_{2}+p \varepsilon_{2}\right)}{\left(s_{1}-p \varepsilon_{1}\right)\left(s_{2}-p \varepsilon_{2}\right)} \exp \left(\frac{2 p \xi_{n} d}{c}\right)-1\right]^{-1}\right\} d p
\end{aligned}
$$

where, with respect to the usual presentation of the force, we have separated a first term behaving like $k_{B} T / d^{3}$ from the other terms with $n \geqslant 1$. In Eq. (15) we have defined

$$
s_{1}=\sqrt{\varepsilon_{1}-1+p^{2}}, \quad s_{2}=\sqrt{\varepsilon_{2}-1+p^{2}},
$$

where $\varepsilon_{1}=\varepsilon_{1}\left(i \xi_{n}\right)$ and $\varepsilon_{2}=\varepsilon_{2}\left(i \xi_{n}\right)$ are the relevant dielectric functions of the two plates, evaluated for imaginary values $\omega=i \xi_{n}$ of the frequency, with $\xi_{n}=2 \pi k_{B} T n / \hbar$. The function $\varepsilon(i \xi)$ is a monotonic and real function of $\xi$, obtained by analytic continuation of the nonmonotonic and complex function $\varepsilon(\omega)$ evaluated for real values of the frequency $\omega$. Furthermore, in Eq. (15) we have introduced the static values of the dielectric functions $\varepsilon_{10}=\varepsilon_{1}(0)$ and $\varepsilon_{20}=\varepsilon_{2}(0)$. Some general properties of these functions will be illustrated in Sec. VI.

The force produced by a surface on a single atom can be easily derived by assuming that one of the two walls (wall 2) is made of a very dilute material (for example a gas) so that one can expand Eq. (15) for small values of $\varepsilon_{2}-1=4 \pi n \alpha$ where $n$ is the density of the gas and $\alpha$ is the corresponding atomic polarizability. The potential $V_{\text {surf }}$ felt by a single atom is then simply given by $V_{\text {surf }}=-F_{\text {surf }} / n$ and can be written in the form [13]

$$
\begin{aligned}
V_{\text {surf }}(d)= & -\frac{k_{B} T \alpha_{0}}{4 d^{3}} \frac{\left(\varepsilon_{0}-1\right)}{\left(\varepsilon_{0}+1\right)}-\frac{K T}{c^{3}} \sum_{n=1}^{\infty} \alpha \xi_{n}^{3} \\
& \times \int_{1}^{\infty} \exp \left(-\frac{2 p \xi_{n} d}{c}\right) f(p) d p,
\end{aligned}
$$

where we have introduced the function $f(p)$ defined by

$$
f(p)=\left[\frac{\left(s_{1}-p\right)}{\left(s_{1}+p\right)}+\left(1-2 p^{2}\right) \frac{\left(s_{1}-p \varepsilon\right)}{\left(s_{1}+p \varepsilon\right)}\right],
$$

in which it is easy to distinguish the reflection Fresnel coefficients $r_{s}(i \xi)$ and $r_{p}(i \xi)$ (see Ref. [1]). In Eqs. (17) and (18) we have omitted the index 1 in the dielectric function characterizing the optical properties of the surface while $\varepsilon_{0}$ and $\alpha_{0}$ are the static values of the dielectric function and of the atomic polarizability, respectively. Equation (17) permits us to describe both the interaction generated by a dielectric and by an ideal metal [in the latter case the function $\varepsilon(\omega)$ behaves like $1 / \omega$ at small $\omega$ and $\left.\left(\varepsilon_{0}-1\right) /\left(\varepsilon_{0}+1\right) \rightarrow 1\right]$.

Equations (15) and (17) have been derived assuming that the whole system is in thermodynamic equilibrium and in particular that its components have the same temperature $T$. In the experiments with Bose-Einstein condensates the atomic gas is cooled down to extremely low temperature, while the substrate remains at room temperature. The result (17) is still expected to be valid in this case, $T$ being the temperature of the substrate. In fact atoms, independently of being at zero or at room temperature, do not contribute to the thermal radiation, their lowest excitation energies being much higher than $k_{B} T$.

It is useful to identify the relevant length scales of the problem (see Fig. 2). A first important length is the thermal wavelength of the photon:

$$
\lambda_{T}=\frac{\hbar c}{k_{B} T},
$$

where $k_{B}$ is the Boltzmann constant. At room temperature $(T=300 \mathrm{~K})$ one has $\lambda_{T}=7.6 \mu \mathrm{m}$. For distances $d$ larger than 


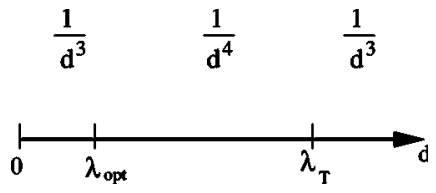

FIG. 2. Relevant length scales $\lambda_{\text {opt }}$ and $\lambda_{T}$ characterizing the generalized van der Waals potential $V_{\text {surf }}$. The power law behavior of $V_{\text {surf }}(d)$ is shown in the appropriate ranges of validity as a function of the distance from the surface.

$\lambda_{T}$ the force is dominated by the thermal fluctuations of the electromagnetic field. In this limit the leading contribution is given by the first term in Eq. (17), due to the occurrence of the exponential factor in the other terms, and the potential takes the characteristic form

$$
V_{\text {surf }}^{T}(d)=-\frac{k_{B} T \alpha_{0}}{4 d^{3}} \frac{\left(\varepsilon_{0}-1\right)}{\left(\varepsilon_{0}+1\right)}
$$

Notice that in this regime only the static value of the dielectric and polarizability functions contribute to the force. It is also worth noticing that in this limit the force is independent of the Planck constant as well as of the velocity of light.

For distances $d$ smaller (or of the same order) than $\lambda_{T}$ the quantum fluctuations of the electromagnetic field become important and the terms of the sum (17) should be taken into account. If $d \ll \lambda_{T}$ and $T$ is sufficiently small, one can replace the sum with an integral (see Sec. IV). In this regime one can introduce a second length scale, hereafter called $\lambda_{\text {opt }}$. This length is related to the relevant wavelengths characterizing the optical properties of the interacting media. One can identify two distinct regimes. For distances $d \ll \lambda_{o p t}$ the potential exhibits the familiar $1 / d^{3}$ van der Waals-London dependence. In the interval $\lambda_{o p t} \ll d \ll \lambda_{T}$ one instead enters the Casimir-Polder regime where the potential decays like $1 / d^{4}$. The possibility of identifying the Casimir-Polder regime depends crucially on the value of the temperature. The temperature should be in fact sufficiently low in order to guarantee the condition $\lambda_{T} \gg \lambda_{\text {opt }}$ (see discussion at the end of Sec. V). Differently from the thermal wavelength, given by the simple expression $\lambda_{T}$, the explicit evaluation of $\lambda_{o p t}$ is not immediate because the optical properties of the media are not characterized by a single frequency (see Sec. VI). In the case of rubidium atoms interacting with a dielectric sapphire substrate we find $\lambda_{\text {opt }} \sim 0.1 \mu \mathrm{m}$.

\section{FORCE AT SHORT DISTANCES: FROM THE van der WAALS-LONDON TO THE CASIMIR-POLDER REGIME}

As anticipated in the preceding section at distances smaller than the thermal wavelength also the terms in the sum (17) contribute to the force and, if $d \ll \lambda_{T}$ and the temperature is sufficiently low, the sum is conveniently replaced by an integral: $\Sigma_{n} \rightarrow\left(\hbar / 2 \pi k_{B} T\right) \int d \xi$. One then finds the useful expression

$$
V_{\text {surf }}^{S R}=-\frac{\hbar}{2 \pi c^{3}} \int_{0}^{\infty} d \xi \int_{1}^{\infty} d p \quad \xi^{3} \alpha \exp \left(-\frac{2 p d \xi}{c}\right) f(p),
$$

for the potential, also called short-range (SR) approximation. It is worth noticing that result (21) does not depend on the value of $T$ [26] and coincides with the zero-temperature limit of the general form (17) of the potential. Equation (21) admits two important limits. For large $d$ one finds the most famous expression

$$
V_{\text {surf }}^{C P}=-\frac{3 \hbar c \alpha_{0}}{8 \pi d^{4}} \frac{\left(\varepsilon_{0}-1\right)}{\left(\varepsilon_{0}+1\right)} \phi\left(\varepsilon_{0}\right),
$$

also know as the Casimir-Polder law, where we have introduced the function [28]

$$
\begin{aligned}
\phi(\varepsilon)= & \frac{(\varepsilon+1)}{(\varepsilon-1)}\left\{\frac{1}{3}+\varepsilon+\frac{4-(\varepsilon+1) \sqrt{\varepsilon}}{2(\varepsilon-1)}\right. \\
& -\frac{\sinh ^{-1} \sqrt{(\varepsilon-1)}\left[1+\varepsilon+2 \varepsilon(\varepsilon-1)^{2}\right]}{2(\varepsilon-1)^{3 / 2}} \\
& \left.+\frac{\varepsilon^{2}\left[\sinh ^{-1} \sqrt{\varepsilon}-\sinh ^{-1}(1 / \sqrt{\varepsilon})\right]}{\sqrt{(\varepsilon+1)}}\right\} .
\end{aligned}
$$

which is equal to 1 in the case of an ideal metal $\left(\varepsilon_{0}=\infty\right)$. For sapphire $\left(\varepsilon_{0}=9.4\right)$ one finds $\phi\left(\varepsilon_{0}\right)=0.8$.

In the opposite limit of small $d$ one instead finds the different law

$$
V_{\text {surf }}^{V L}=-\frac{\hbar}{4 \pi d^{3}} \int_{0}^{\infty} \alpha(i \xi) \frac{\varepsilon(i \xi)-1}{\varepsilon(i \xi)+1} d \xi,
$$

also known as the van der Waals-London (VL) interaction. The coefficient characterizing this force is fixed by the optical properties of the media. The matching between the two laws (22) and (24) provides a useful estimate of the optical length $\lambda_{\text {opt }}$ according to

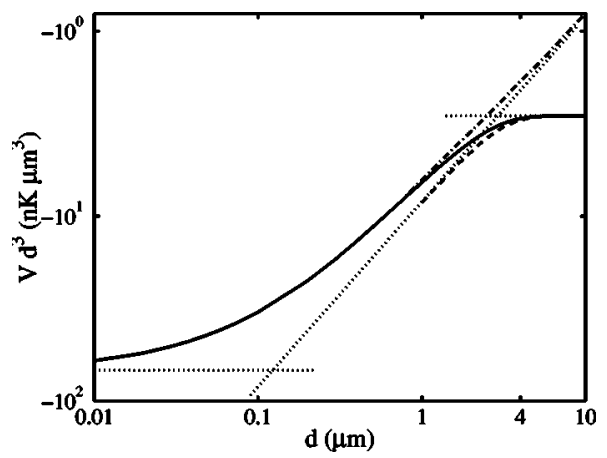

FIG. 3. The atom-surface potential is shown using the exact formula of Eq. (17) (solid line), the short-range approximation (21) (dash-dotted line), and the static approximation (26) (dashed line). The asymptotic van der Waals-London $\left(\sim 1 / d^{3}\right)$, Casimir-Polder $\left(\sim 1 / d^{4}\right)$, and high- $T\left(\sim 1 / d^{4}\right)$ potentials are also shown (dotted lines). The curves were obtained for a sapphire substrate at $300 \mathrm{~K}$ and for ${ }^{87} \mathrm{Rb}$ atoms in the condensate. 


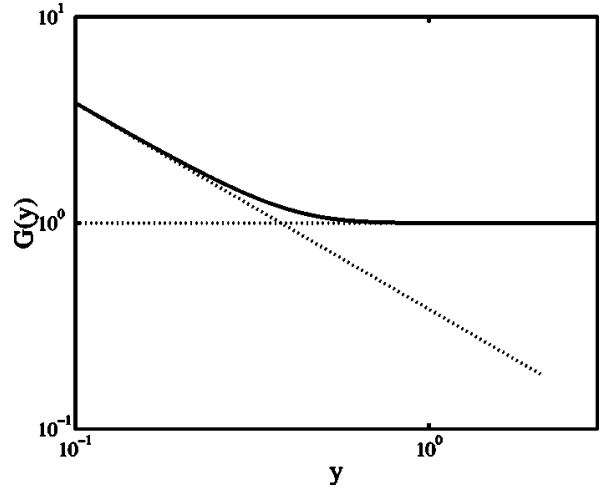

FIG. 4. The function $G(y)$ (solid line) is shown as a function of the scaling variable $y=d / \lambda_{T}$ together with its asymptotic behaviors (dotted lines). The function was calculated for the sapphire substrate (see text).

$$
\lambda_{\text {opt }}=\frac{3 c \alpha_{0}}{2} \frac{\left(\varepsilon_{0}-1\right)}{\varepsilon_{0}+1} \phi\left(\varepsilon_{0}\right)\left[\int_{0}^{\infty} \alpha(i \xi) \frac{\varepsilon(i \xi)-1}{\varepsilon(i \xi)+1} d \xi\right]^{-1} .
$$

In Fig. 3 we show the potential (21) (dash-dotted line) calulated using the dielectric functions of rubidium atom and of sapphire. The asymptotic laws $V_{\text {surf }}^{V L}=-68.1 / d^{3} \mathrm{nK}\left(\mu \mathrm{m}^{3}\right)$ for the van der Waals [see Eq. (24)] and $V_{\text {surf }}^{C P}$ $=-8.34 / d^{4} \mathrm{nK}\left(\mu \mathrm{m}^{4}\right)$ for the Casimir-Polder [see Eq. (22)] potentials, as well as the asymptotic thermal law $V_{\text {surf }}^{T}$ $=-2.86 / d^{3} \mathrm{nK}\left(\mu \mathrm{m}^{3}\right)$ evaluated at $T=300 \mathrm{~K}$ [see Eq. (20)], are also shown ( $d$ is here expressed in microns). It is worth noticing that the potential (21) approaches the asymptotic Casimir-Polder force only at distances significantly larger than $\lambda_{\text {opt }}$.

\section{FORCE AT LARGE DISTANCES AND THE STATIC APPROXIMATION: QUANTUM VS THERMAL EFFECTS}

At large distances from the wall the potential (17) can be safely evaluated in the so called static approximation (SA)

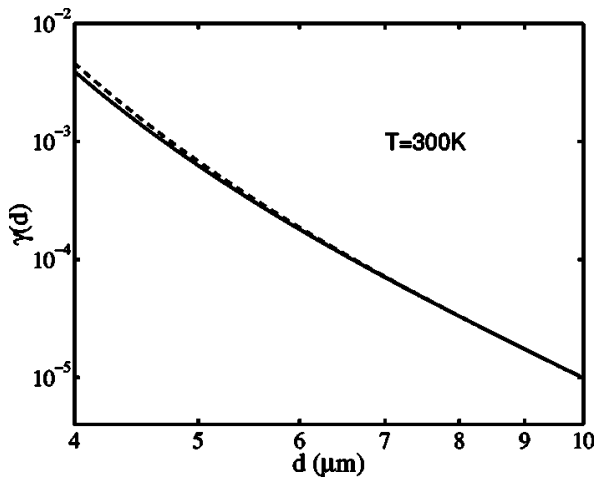

FIG. 5. Fractional frequency shift (32) of the center-of-mass oscillation (solid line) as a function of the distance from the surface, compared with the prediction of the static approximation (dashed line). The curves were obtained for a sapphire substrate at $300 \mathrm{~K}$ and for ${ }^{87} \mathrm{Rb}$ atoms in the condensate. The unperturbed frequency was $\omega_{z} / 2 \pi=220 \mathrm{~Hz}$ while the radius of the condensate was $R_{z}$ $=2.5 \mu \mathrm{m}$. which consists of replacing the dielectric functions $\varepsilon$ and $\alpha$ with their static values $\varepsilon_{0}$ and $\alpha_{0}$, respectively. In the static approximation the potential generated by the surface can be written in the useful form

$$
V_{\text {surf }}^{S A}(d)=-\frac{K T \alpha_{0}}{4 d^{3}} \frac{\left(\varepsilon_{0}-1\right)}{\left(\varepsilon_{0}+1\right)} G(y),
$$

where we have introduced the relevant scaling variable

$$
y=\frac{d}{\lambda_{T}}
$$

and the function

$$
G(y)=\left[1+32 \pi^{3} \frac{\left(\varepsilon_{0}+1\right)}{\left(\varepsilon_{0}-1\right)} \int_{1}^{\infty} y^{3} g(4 \pi p \quad y) f_{0}(p) d p\right] .
$$

In Eq. (28), $f_{0}(p)$ is the static limit of Eq. (18), obtained by replacing $\varepsilon$ with $\varepsilon_{0}$, while the function $g$ is defined by

$$
g(a) \equiv \sum_{n=1}^{\infty} n^{3} e^{-a n}=\frac{e^{-a}\left[1+4 e^{-a}+e^{-2 a}\right]}{\left(1-e^{-a}\right)^{4}} .
$$

The asymptotic behaviors of the function $G(y)$ are well established: for $y \rightarrow 0$ one finds $G(y) \rightarrow 3 \phi\left(\varepsilon_{0}\right) /(2 \pi y)$. Vice versa, for $y \rightarrow \infty$ one finds $G(y) \rightarrow 1$. For a fixed value of $T$ the two limits apply, respectively, to distances larger and smaller than the thermal wavelength (19). It then follows that the static approximation (26) provides the transition from the Casimir-Polder law (22) holding for $\lambda_{\text {opt }} \ll d \ll \lambda_{T}$, to the thermal law (20) holding for $d \gg \lambda_{T}$. In Fig. 4 we show the scaling function $G(y)$ together with the two asymptotic limits $y \rightarrow 0$ and $y \rightarrow \infty$ in the case of sapphire where we have used the value $\varepsilon_{0}=9.4$. The function $G(y)$ depends rather smoothly on the actual value of the static dielectric function, provided the value $\varepsilon_{0}$ is significantly larger than 1 .

In Fig. 3 we compare the prediction for the potential given by the static approximation with the exact result evaluated starting from Eq. (17). The comparison shows that for distances larger than $\sim 2 \mu \mathrm{m}$ the static approximation is rather accurate. This explains why the predictions of this approximation for the frequency shifts are also accurate (see Figs. 5 and 6).

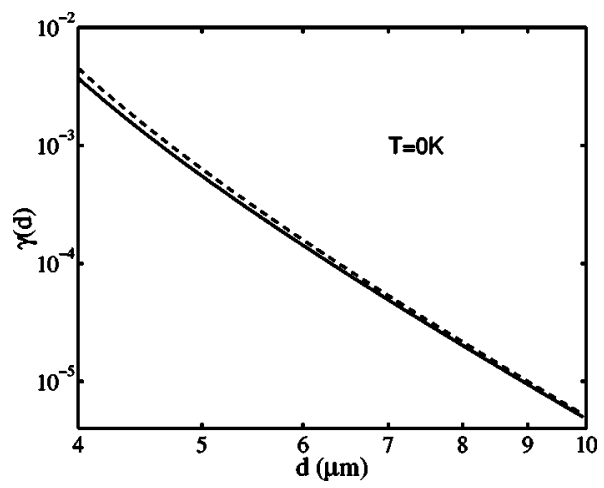

FIG. 6. Same as in Fig. 5, at $T=0 \mathrm{~K}$. 


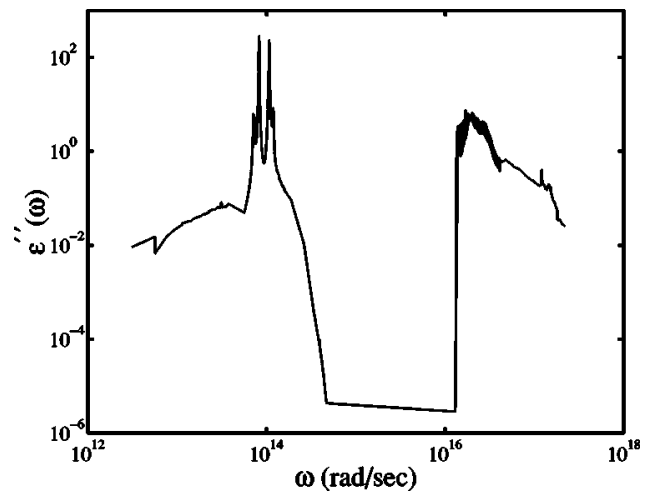

FIG. 7. Imaginary part of the sapphire dielectric function $\varepsilon^{\prime \prime}(\omega)$ on the real axis of frequences.

At shorter distances the static approximation fails and one should instead use the short-range description developed in Sec. IV. If $\lambda_{T} \gg \lambda_{o p t}$, the long-range description based on the static approximation matches the short-range description of Sec. IV in the Casimir-Polder regime (22). In this case the matching procedure completes the determination of the potential at all distances. If instead the condition $\lambda_{T} \gg \lambda_{\text {opt }}$ is not well satisfied, one should calculate explicitly the potential using the general expression (17) and the potential will not exhibit the intermediate Casimir-Polder behavior (22). For example, in the case of Fig. 3, where the potential is calculated at $T=300 \mathrm{~K}$, the matching between the two curves is not very good and consequently the system never exhibits the Casimir-Polder behavior [27].

\section{OPTICAL PROPERTIES OF THE ATOM AND OF THE SUBSTRATE}

The functions $\varepsilon(i \xi)$ and $\alpha(i \xi)$, which are crucial ingredients for the calculation of the potential generated by the surface, obey the Kramers-Kronig relations

$$
\varepsilon\left(i \quad \xi_{n}\right)=1+\frac{2}{\pi} \int_{0}^{\infty} \frac{\omega \varepsilon^{\prime \prime}(\omega)}{\omega^{2}+\xi_{n}^{2}} d \omega,
$$

where $\varepsilon^{\prime \prime}$ is the imaginary part of the dielectric function $\varepsilon$ $=\varepsilon^{\prime}+i \varepsilon^{\prime \prime}$ evaluated on the real axis. For the polarizability one has the analogous relationship

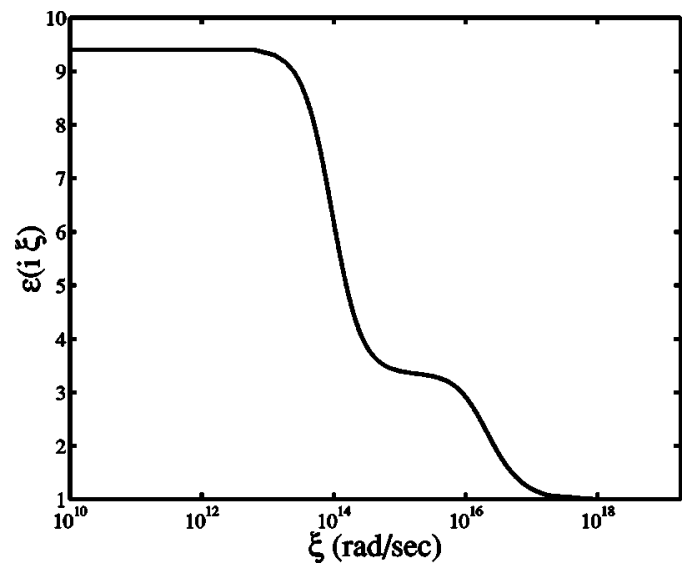

FIG. 8. Sapphire dielectric function $\varepsilon(i \xi)$ evaluated on the imaginary axis.

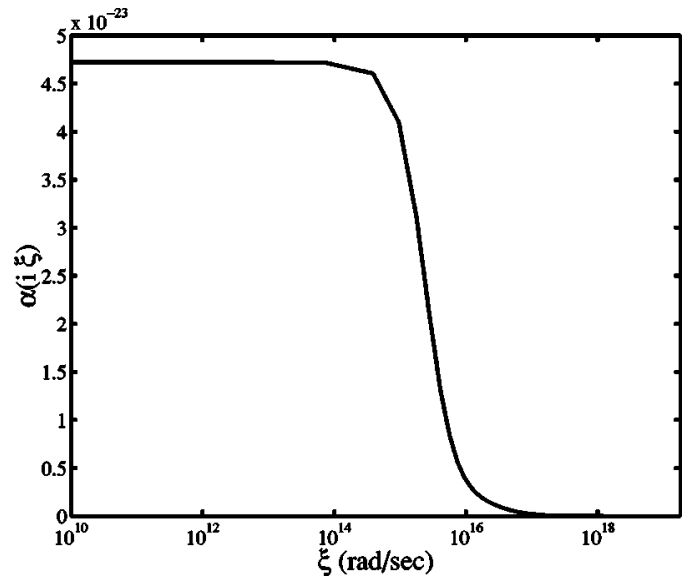

FIG. 9. Polarizability function $\alpha(i \xi)$ (in $\mathrm{cm}^{3}$ ) for ${ }^{87} \mathrm{Rb}$ atoms evaluated on the imaginary axis.

$$
\alpha\left(i \quad \xi_{n}\right)=\frac{2}{\pi} \int_{0}^{\infty} \frac{\omega \alpha^{\prime \prime}(\omega)}{\omega^{2}+\xi_{n}^{2}} d \omega,
$$

where $\alpha^{\prime \prime}$ is the imaginary part of the atomic polarizability $\alpha=\alpha^{\prime}+i \alpha^{\prime \prime}$.

The imaginary part of the dielectric function can be related to the measurable real and imaginary parts of the refraction index $n(\omega)=n^{\prime}(\omega)+i n^{\prime \prime}(\omega)$ according the equation $\varepsilon^{\prime \prime}(\omega)=2 n^{\prime}(\omega) n^{\prime \prime}(\omega)$. The values of $\varepsilon^{\prime \prime}(\omega)$ (data taken from [30]) and $\varepsilon(i \xi)$ for sapphire are reported in Figs. 7 and 8. From the optical point of view, crystalline sapphire is a uniaxial crystal and one should distinguish between the ordinary and extraordinary refraction indices. Since the optical properties for sapphire are not completely known, in our calculations we have used only the data for ordinary waves. We expect that this approximation will not significantly affect the analysis in both crystalline and melted sapphires. In Fig. 9 we report the corresponding values for $\alpha(i \xi)$ for ${ }^{87} \mathrm{Rb}$ [29]. The static value of the polarizability is $\alpha_{0}=47.3$ $\times 10^{-24} \mathrm{~cm}^{3}$. Figure 7 shows that the optical properties of sapphire are rather complex so that the actual value of $\lambda_{\text {opt }}$ governing the transition from the van der Waals-London to the Casimir-Polder regimes cannot be simply inferred from the form of $\varepsilon^{\prime \prime}$, but requires the explicit calculation of the integral (25). The typical "two-plateau" behavior exhibited by $\varepsilon(i \xi)$ in solid dielectrics is the consequence of the concentration of the strength $\varepsilon^{\prime \prime}(\omega)$ in two distinct regions of frequencies (see Figs. 7 and 8). This behavior is absent in the atomic polarizability (see Fig. 9). Using Eq. (25) it is possible to calculate the value of $\lambda_{\text {opt }}$. In the case of the sapphire substrate interacting with rubidium atoms we find $\lambda_{\text {opt }}$ $=0.1 \mu \mathrm{m}$.

\section{RESULTS FOR THE SHIFT OF THE CENTER-OF-MASS OSCILLATION}

We are now ready to calculate the shifts of the center-ofmass frequencies of the condensate caused by the force generated by the surface. We will consider a condensate of finite Thomas-Fermi radius $R_{z}$ located a distance $d$ from the sur- 


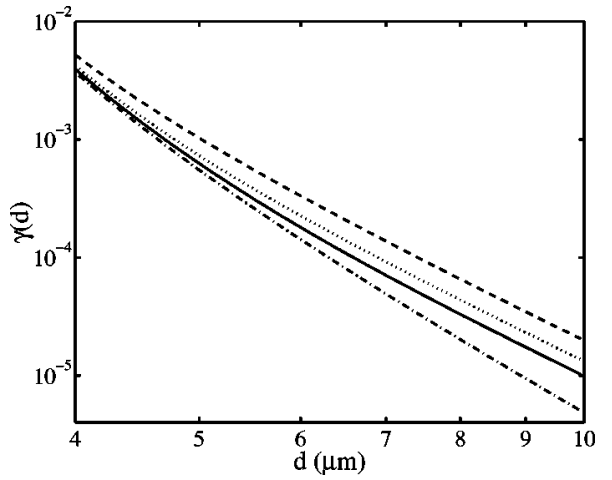

FIG. 10. Relative frequency shift (32) of the center-of-mass oscillation calculated at $T=0 \mathrm{~K}$ (dash-dotted line), $T=300 \mathrm{~K}$ (solid line), $T=400 \mathrm{~K}$ (dotted line), and at $T=600 \mathrm{~K}$ (dashed line). The curves were obtained for a sapphire substrate and for ${ }^{87} \mathrm{Rb}$ atoms in the condensate. The unperturbed frequency was $\omega_{z} / 2 \pi=220 \mathrm{~Hz}$ while the radius of the condensate was $R_{z}=2.5 \mu \mathrm{m}$.

face (see Fig. 1). We will evaluate the fractional frequency shift

$$
\gamma=\frac{\omega_{z}-\omega_{\mathrm{c} . \mathrm{m} .}}{\omega_{z}}
$$

with $\omega_{\text {c.m. }}$ given by Eq. (8), as a function of the distance $d$ at different temperatures. As discussed in the preceding sections, the behavior of the potential $V_{\text {surf }}$ at short distances is temperature independent and coincides with the zerotemperature value. Conversely, at distances of the order of the thermal wavelength or larger, the potential exhibits an important temperature dependence. This behavior is reflected in the shift of the center-of-mass frequency of the condensate for which we provide our predictions in Fig. 10 at different temperatures. The curves of this figure have been calculated using the general expression (17) for the potential. In the calculation we have used the values $\omega / 2 \pi=220 \mathrm{~Hz}$ for the harmonic trapping frequency along the $z$ th direction and $R_{z}$ $=2.5 \mu \mathrm{m}$ for the $z$ th radius of the condensate. These are typical values employed in current experiments with BoseEinstein condensates. We have used a sapphire substrate and the condensate is made of ${ }^{87} \mathrm{Rb}$ atoms. Our predictions show that at distances $d \sim 4-8 \mu \mathrm{m}$, where the thermal effects start becoming important at room temperature, the frequency shifts are of the order of $10^{-4}$. These results are promising in view of the possibility of systematic measurements of the interplay between quantum and thermal effects in the generalized van der Waals forces.

It is also interesting to compare the predictions for the shifts of the collective frequencies with the results obtained using the static approximation, holding at large distances (see Sec. V). The comparison (see Figs. 5 and 6) shows that the static approximation (26) provides an accurate decription of the shifts for distances larger than $4 \mu \mathrm{m}$, even at zero temperature. At shorter distances the deviations become more important. One should actually notice that at short distances the width of the condensate plays an important role amplifying the effects of the short-range component of the potential.
Let us finally discuss the effects of nonlinearities on the frequency shift. If the amplitude of the oscillation is not small, additional corrections due to the external force should be taken into account (see Sec. II). The corresponding corrections can be estimated using the result (14). For example, by choosing $a=0.5 \mu \mathrm{m}$ and making the same choices for the other parameters $\left(R_{z}=2.5\right.$ and $\left.\omega_{z} / 2 \pi=220 \mathrm{~Hz}\right)$, we find that the prediction for the frequency shift (32) is increased, in absolute value, by $\sim 20 \%$ at $d=6 \mu \mathrm{m}$. This result shows that for larger choices of the amplitude of the oscillation and for smaller values of $d$ the effects of nonlinearity can become very important. In general they should be calculated starting directly from Eqs. (12) and (13).

\section{CONCLUSIONS}

In this work we have applied Lifshitz theory of generalized van der Waals interactions to investigate the effect of a substrate on the collective oscillations of a trapped quantum gas. We have first developed the general theory for the frequency shifts of the center-of-mass oscillation caused by a perturbative force of general form. We have hence characterized the potential generated by the surface identifying various relevant regimes. A first approximation, holding at short distances $d$ from the surface, permits one to describe the transition from the van der Waals-London $1 / d^{3}$ law to the Casimir-Polder $1 / d^{4}$ regime characterized by quantum and relativistic effects. A second approximation, holding at larger distances, permits one to describe the transition from the Casimir-Polder law to the large distance $k_{B} T / d^{3}$ behavior, dominated by the thermal fluctuations of the electromagnetic field. We have also identified the relevant length scales of the problem and explored the matching conditions for the various approximations. Due to the finite size of the condensate, the calculation of the frequency shifts requires a numerical integration, although analytical expressions can be obtained in some limiting cases. The calculation requires explicit knowledge of the optical properties of the interacting media and in particular the dielctric function of the susbstrate and the atomic polarizability of the atoms in the condensate. We have also exploited the effects of nonlinearity and shown that, due to the finite width of the condensate, they can provide large corrections to the frequency shifts and should be consequently taken into account in the comparison with future experiments. We have finally derived (see the Appendix) results for the frequency shifts of the radial breathing mode.

Our predictions are rather promising and suggest that Bose-Einstein condensates can become efficient sensors of very weak forces. In particular the oscillation of the condensate permits one to explore the interplay between quantum and thermal fluctuations of the electromagnetic field in the relevant region of $4-8 \mu \mathrm{m}$. For such distances the relative shifts are predicted to be of the order of $10^{-4}$ and are hence measurable experimentally.

\section{ACKNOWLEDGMENTS}

This work was stimulated by insightful discussions with Eric Cornell. It is a pleasure to thank him as well as John 
Obrecht, Jeffrey McGuirk, and David Murray Harber for many useful comments. We would also like to thank J.F. Babb for sending us the calculated values of $\alpha(i \xi)$ for rubidium.

\section{APPENDIX: FREQUENCY SHIFT OF THE RADIAL BREATHING MODE}

The radial breathing oscillation in elongated traps is a fundamental mode exhibited by Bose-Einstein condensates. For an interacting Bose-Einstein condensate, harmonically trapped in two dimensions, this mode exactly occurs at twice the trapping radial frequency (we assume here symmetric trapping: $\omega_{x}=\omega_{z} \equiv \omega_{\perp}$ ), irrespective of the amplitude of the oscillation, number of atoms, and value of the scattering length [31]. In a 3D configuration this mode can be realized in the limit of a very elongated axisymmetric trap $\left(\omega_{y}\right.$ $\left.\ll \omega_{\perp}\right)$ corresponding, in the geometry of Fig. 1, to a condensate very elongated along the direction orthogonal to the figure. The radial breathing oscillation has been measured with high accuracy in [32].

The radial breathing mode can be described by deriving the time-dependent equations for the average square radius $\left\langle x^{2}+z^{2}\right\rangle$ within Gross-Pitaevskii theory [17] in 2D. After some straightforward algebra one finds the equation

$$
\frac{d^{2}}{d t^{2}}\left\langle x^{2}+z^{2}\right\rangle=-\frac{2}{m}\left\langle\mathbf{r} \cdot \partial_{\mathbf{r}} V\right\rangle+\frac{2}{m^{2}}\left\langle p_{x}^{2}+p_{z}^{2}\right\rangle+\frac{4}{m}\left\langle V_{2-\text { body }}\right\rangle,
$$

where $V_{2-b o d y}$ is the average values of the mean-field interaction energy and $V$ is the external potential. By introducing the energy per particle, $E / N=\langle V\rangle+\left\langle p_{x}^{2}+p_{z}^{2}\right\rangle / 2 m+\left\langle V_{2-b o d y}\right\rangle$, which is conserved in time, and separating in $V$ the axisymmetric harmonic potential $V_{h o}$ from the surface potential $V_{\text {surf }}$, Eq. (A1) can be rewritten as

$$
\frac{d^{2}}{d t^{2}}\left\langle x^{2}+z^{2}\right\rangle=\frac{4}{m} E-4 \omega_{\perp}^{2}\left\langle x^{2}+z^{2}\right\rangle-\frac{4}{m}\left\langle V_{\text {surf }}\right\rangle-\frac{2}{m}\left\langle z \partial_{z} V_{\text {surf }}\right\rangle .
$$

In the absence of the surface perturbation $\left(V_{\text {surf }}=0\right)$, Eq. (A2) gives oscillating solutions with $\omega=2 \omega_{\perp}$.

The effect of the perturbation is simply calculated noticing that the unperturbed solution corresponds to a scaling transformation where the density varies as $n(x, y, t)$ $=\alpha^{2} n_{0}(x / \alpha, y / \alpha)$ and $n_{0}$ is the equilibrium density distribution. The time-dependent scaling parameter $\alpha$ fixes the value of the square radius according to $\left\langle x^{2}+z^{2}\right\rangle=\alpha^{2}\left\langle x^{2}+z^{2}\right\rangle_{0}$. Using the scaling transformation to evaluate the integrals $\left\langle V_{\text {surf }}\right\rangle$ $=\int d \mathbf{r} n(x, y, t) V_{\text {surf }}(z)$ and $\left\langle z \partial_{z} V_{\text {surf }}\right\rangle=\int d \mathbf{r} n(x, y, t) z \partial_{z} V_{\text {surf }}(z)$ one can rewrite Eq. (A2) as a simple equation for the parameter $\alpha$. In the following we will be interested in the limit of small-amplitude oscillations. By expanding the integrals up to first order in $(\alpha-1)$, one finally obtains the result

$$
\begin{aligned}
\omega_{B}^{2}= & 4 \omega_{\perp}^{2}+\frac{1}{m\left\langle x^{2}+z^{2}\right\rangle}\left[\int_{-R_{z}}^{R_{z}} n_{0}^{z} z \partial_{z} z \partial_{z} V_{\text {surf }} d z\right. \\
& \left.+2 \int_{-R_{z}}^{R_{z}} n_{0}^{z} z \partial_{z} V_{\text {surf }} d z\right]
\end{aligned}
$$

for the frequency shift of the radial breathing $(B)$ mode, where $n_{0}^{z}$ is the $1 \mathrm{D}$ column density (7). Similarly to the case of the shift of the center-of-mass frequency [see Eq. (32)], also the result (A3) is exact up to first-order corrections in the perturbation. In fact the radial scaling ansatz is an exact solution of the Gross-Pitaevskii equation in 2D. It is also a good approximation in 3D if the harmonic trap is sufficiently elongated. The result (A3) for the radial breathing mode may provide a further tool to investigate the effects of the generalized van der Waals force on a Bose-Einstein condensate.
[1] J. F. Babb, G. L. Klimchitskaya, and V. M. Mostepanenko, Phys. Rev. A 70, 042901 (2004).

[2] K. A. Milton, J. Phys. A 37, R209 (2004).

[3] H. B. Chan et al., Phys. Rev. Lett. 87, 211801 (2001).

[4] S. Dimopoulos and A. A. Geraci, Phys. Rev. D 68, 124021 (2003)

[5] M. Bordag, U. Mohideen, and V. M. Mostepanenko, Phys. Rep. 353, 1 (2001).

[6] Y. J. Lin, I. Teper, C. Chin, and V. Vuletic, Phys. Rev. Lett. 92, 050404 (2004).

[7] A. E. Leanhardt et al., Phys. Rev. Lett. 90, 100404 (2003).

[8] D. M. Harber, J. M. McGuirk, J. M. Obrecht, and E. A. Cornell, J. Low Temp. Phys. 133, 229 (2003); J. M. McGuirk, D. M. Harber, J. M. Obrecht, and E. A. Cornell, e-print cond-mat/ 0403254

[9] F. London, Z. Phys. 60, 491 (1930).

[10] H. B. G. Casimir and D. Polder, Phys. Rev. 73, 360 (1948).

[11] It is worth pointing out that the interaction between macroscopic bodies is not in general the result of a simple summa- tion of the interatomic force. Additivity is guaranteed only in the case of rarefied gases.

[12] E. M. Lifshitz, Sov. Phys. JETP 3, 977 (1957).

[13] I. E. Dzyaloshinskii, E. M. Lifshitz, and L. P. Pitaevskii, Adv. Phys. 38, 165 (1961). For a more recent derivation of the Lifshitz theory see P. Milonni, The Quantum Vacuum: An Introduction to Quantum Electrodynamics (Academic Press, Boston, 1994) and K. A. Milton, The Casimir Effect: Physical Manifestations of Zero-Point Energy (World Scientific, Singapore, 2001).

[14] F. Dalfovo, S. Giorgini, L. P. Pitaevskii, and S. Stringari, Rev. Mod. Phys. 71, 463 (1999).

[15] A. J. Leggett, Rev. Mod. Phys. 73, 307 (2001).

[16] C. J. Pethick and H. Smith, Bose-Einstein Condensation in Dilute Bose Gases (Cambridge University Press, Cambridge, England, 2002).

[17] L. P. Pitaevskii and S. Stringari, Bose-Einstein Condensation (Oxford University Press, Oxford, 2003).

[18] M. R. Andrews et al., Science 275, 637 (1997). 
[19] C. Orzel et al., Science 291, 2386 (2001).

[20] S. Stringari, Phys. Rev. Lett. 77, 2360 (1996).

[21] D. S. Jin et al., Phys. Rev. Lett. 77, 420 (1996).

[22] D. M. Stamper-Kurn et al., Phys. Rev. Lett. 81, 500 (1998).

[23] W. Kohn, Phys. Rev. 123, 1242 (1961).

[24] Due the external force generated by the wall, the center-ofmass position of the gas at rest does not coincide with the center of the harmonic trap. This sag effect is, however, very small and can be safely ignored.

[25] In the case of an ideal degenerate Fermi gas confined in a harmonic trap one instead finds the result $n_{0}^{z}(z)$ $=\left(16 / 5 \pi R_{z}\right)\left(1-z^{2} / R_{z}^{2}\right)^{5 / 2}$, where $R_{z}$ is the corresponding Thomas-Fermi radius, determined by the Fermi energy $E_{F}$ $=\hbar \omega_{h o}(6 N)^{1 / 3}$ according to the formula $E_{F}=(1 / 2) m \omega_{z}^{2} R_{z}^{2}$. Here $N$ is the number of trapped atoms of a single spin species.
[26] Temperature can still affect the value of the dielectric and polarizability functions, but this effect is small and has been ignored in the present work.

[27] In this paper we will not discuss the opposite limit $\lambda_{T} \ll \lambda_{\text {opt }}$ which is never reached in realistic conditions.

[28] B. V. Deryagin, I. I. Abrikosova, and E. M. Lifshitz, Q. Rev., Chem. Soc. 10, 295 (1958); Usp. Fiz. Nauk 64, 493 (1958).

[29] J. F. Babb (private communication).

[30] W. J. Tropf and M. E. Thomas, in Handbook of Optical Constants of Solids, edited by E. Palik (Academic Press, New York, 1998), Vol. III.

[31] L. P. Pitaevskii, Phys. Lett. A 221, 14 (1996); Yu. Kagan, E. L. Surkov, and G. V. Shlyapnikov, Phys. Rev. A 54, R1753 (1996).

[32] F. Chevy et al., Phys. Rev. Lett. 88, 250402 (2002). 